Georgian Mathematical Journal

Volume 12 (2005), Number 2, 337-348

\title{
SOME REMARKS CONCERNING JONES EIGENFREQUENCIES AND JONES MODES
}

\author{
DAVID NATROSHVILI, GURAM SADUNISHVILI, AND IRINE SIGUA
}

\begin{abstract}
Three-dimensional fluid-solid interaction problems with regard for thermal stresses are considered. An elastic structure is assumed to be a bounded homogeneous isotropic body occupying a domain $\Omega^{+} \subset \mathbb{R}^{3}$, where the thermoelastic four dimensional field is defined, while in the unbounded exterior domain $\Omega^{-}=\mathbb{R}^{3} \backslash \overline{\Omega^{+}}$there is defined the scalar (acoustic pressure) field. These two fields satisfy the differential equations of steady state oscillations in the corresponding domains along with the transmission conditions of special type on the interface $\partial \Omega^{ \pm}$. We show that uniqueness of solutions strongly depends on the geometry of the boundary $\partial \Omega^{ \pm}$. In particular, we prove that for the corresponding homogeneous transmission problem for a ball there exist infinitely many exceptional values of the oscillation parameter (Jones eigenfrequencies). The corresponding eigenvectors (Jones modes) are written explicitly. On the other hand, we show that if the boundary surface $\partial \Omega^{+}$contains two flat, non-parallel sub-manifolds then there are no Jones eigenfrequencies for such domains.
\end{abstract}

2000 Mathematics Subject Classification: 74F10, 74F05.

Key words and phrases: Elasticity, thermoelasticity, fluid-solid interaction, Jones eigenfrequencies, Jones modes.

\section{INTRODUCTION}

Problems connected with the interaction between vector fields of different dimension have received much attention in the mathematical and engineering scientific literature and have been intensively investigated for the past years. They arise in many physical and mechanical models describing the interaction of two different media where the whole process is characterized by a vectorfunction of dimension $k$ in one medium and by a vector-function of dimension $n$ in another one (for example, the fluid-structure interaction when a streamlined body is an elastic obstacle, the scattering of acoustic and electromagnetic waves by an elastic obstacle, the interaction between an elastic body and seismic waves, etc.).

Quite many authors have considered in detail time-harmonic dependent fluidsolid interaction problems involving the so-called frequency parameter $\omega$. Exhaustive information in this direction can be found in [1], [3], [4], [5], [6], [9], [10], [12], [14], [17] (see also the references therein).

Here we consider three-dimensional fluid-solid interaction problems with regard for thermal stresses. An elastic structure is assumed to be a bounded 
homogeneous isotropic body occupying a domain $\Omega^{+} \subset \mathbb{R}^{3}$, where the thermoelastic four-dimensional field is defined, while in the unbounded exterior domain $\Omega^{-}=\mathbb{R}^{3} \backslash \overline{\Omega^{+}}$the scalar (acoustic pressure) field is defined. These two fields satisfy the differential equations of steady state oscillations in the corresponding domains along with transmission conditions of a special type on the interface $\partial \Omega^{ \pm}$.

In general, the corresponding transmission problem is not uniquely solvable for all values of the oscillation parameter $\omega$. We remark that the uniqueness of solutions plays a crucial role in many applications (e.g., in inverse fluid solidinteraction problems related to shape identification problems). Till now, almost nothing has been known of exceptional values (the so-called Jones eigenfrequencies) of the oscillation parameter $\omega$ for which the homogeneous transmission problem has nontrivial solutions (Jones modes).

In this paper we show that the uniqueness of solutions strongly depends on the geometry of the boundary $\partial \Omega^{ \pm}$. We prove that for the corresponding homogeneous transmission problem for a ball there exist infinitely many Jones eigenfrequencies and Jones modes. We have obtained an explicit equation (in terms of Bessel functions) for Jones eigenfrequencies. The corresponding Jones modes are written explicitly. On the other hand, we have established that if the boundary surface $\partial \Omega^{+}$contains two flat, non-parallel sub-manifolds then there exist no Jones eigenfrequencies for such domains.

\section{Formulation of the Problem. General Uniqueness Results}

Let $\Omega^{+} \subset \mathbb{R}^{3}$ be a bounded domain with the boundary $\partial \Omega^{+}=S, \overline{\Omega^{+}}=$ $\Omega^{+} \cup S$, and let $\Omega^{-}=\mathbb{R}^{3} \backslash \overline{\Omega^{+}}$. We assume that the domain $\overline{\Omega^{+}}$is filled up by a homogeneous isotropic material. The thermoelastic oscillation field is defined in $\overline{\Omega^{+}}$and is described by the displacement vector $u=\left(u_{1}, u_{2}, u_{3}\right)^{\top}$ and the temperature distribution function $u_{4}$ (here and in what follows the symbol ${ }^{\top}$ denotes transposition). In the domain $\Omega^{-}$the oscillation field is described by the wave (metaharmonic) function $w$.

The interaction of these two fields is mathematically described by the following transmission problem (see, e.g., [5] and the references therein).

Problem A. Find the displacement vector $u$ and the temperature distribution function $u_{4}$ in $\Omega^{+}$, and the metaharmonic function $w$ in $\Omega^{-}$which satisfy

i) the differential equations of coupled thermoelasticity in $\Omega^{+}$

$$
\left\{\begin{array}{l}
\mu \Delta u+(\lambda+\mu) \operatorname{grad} \operatorname{div} u+\varrho \omega^{2} u-\gamma \operatorname{grad} u_{4}=0 \\
\Delta u_{4}+\frac{i \omega}{\varkappa} u_{4}+i \omega \eta \operatorname{div} u=0
\end{array}\right.
$$

and the Helmholtz equation in $\Omega^{-}$

$$
\left(\Delta+\varrho_{1} \omega^{2}\right) w=0,
$$


ii) the boundary transmission conditions on $S$

$$
\begin{aligned}
& \{P U\}^{+}=d_{2}\{w\}^{-} n+f, \\
& \{n \cdot u\}^{+}=d_{1}\left\{\partial_{n} w\right\}^{-}+f_{4}, \\
& \left\{\alpha \partial_{n} u_{4}+\beta u_{4}\right\}^{+}=f_{5},
\end{aligned}
$$

where $n$ is the outward unit normal vector to $S$, the symbols $\{\cdot\}^{ \pm}$denote the limits on $S$ from $\Omega^{ \pm}, \partial_{n}$ is the usual directional differentiation along the normal vector $n, \omega>0$ is a frequency, $U=\left(u_{1}, u_{2}, u_{3}, u_{4}\right)^{\top}, P U$ is the thermo-stress vector [11]

$$
P U=P\left(\partial_{x}, n(x)\right) U(x):=2 \mu \partial_{n} u+\lambda n \operatorname{div} u+\mu[n \times \operatorname{rot} u]-\gamma u_{4} n ;
$$

$\partial_{x}=\left(\partial_{1}, \partial_{2}, \partial_{3}\right), \partial_{j}=\partial / \partial x_{j}, j=1,2,3$; for the vectors $a=\left(a_{1}, a_{2}, a_{3}\right)$ and $b=\left(b_{1}, b_{2}, b_{3}\right)$ the symbols $a \cdot b$ and $a \times b$ denote the usual inner product and the cross product, respectively; $d_{1}, d_{2}, \alpha$, and $\beta$ are some complex constants, while $f=\left(f_{1}, f_{2}, f_{3}\right)^{\top}, f_{4}$ and $f_{5}$ are given functions on $S ; \lambda$ and $\mu$ are the Lamé constants, $\varrho_{1}, \varrho, \gamma, \varkappa$, and $\eta$ are positive constants characterizing the thermomechanical properties of materials (see, e.g., [16], [11], [5]-[8], [2], [4]).

Moreover, we provide $u_{j} \in C^{1}\left(\overline{\Omega^{+}}\right) \bigcap C^{2}\left(\Omega^{+}\right), w \in C^{1}\left(\overline{\Omega^{-}}\right) \bigcap C^{2}\left(\Omega^{-}\right)$, and, in addition, $w$ satisfies the Sommerfeld radiation condition at infinity

$$
\frac{\partial w(x)}{\partial r}-i \sqrt{\varrho_{1}} \omega w(x)=O\left(r^{-2}\right) \quad \text { as } \quad r \rightarrow+\infty, \quad r=|x| .
$$

In what follows we will study the uniqueness question for the homogeneous Problem A. To this end, let us introduce the following notation.

Denote by $J\left(\Omega^{+}\right)$the set of all values of the oscillation parameter $\omega$ for which the BVP

$$
\begin{aligned}
& \Delta u+\omega_{3}^{2} u=0 \quad \text { in } \quad \Omega^{+}, \quad u=\left(u_{1}, u_{2}, u_{3}\right)^{\top}, \quad \omega_{3}^{2}=\frac{\varrho \omega^{2}}{\mu}, \\
& \operatorname{div} u=0 \quad \text { in } \Omega^{+}, \\
& \left\{T\left(\partial_{x}, n(x)\right) u(x)\right\}^{+}:=\left\{2 \mu \partial_{n} u(x)+\mu[n(x) \times u(x)]\right\}^{+}=0 \quad \text { on } \partial \Omega^{+}, \\
& \{n(x) \cdot u(x)\}^{+}=0 \quad \text { on } \partial \Omega^{+} .
\end{aligned}
$$

possesses a nontrivial solution. Such values of the parameter $\omega$ are called Jones eigenfrequencies. To each $\omega \in J\left(\Omega^{+}\right)$there correspond only finitely many linearly independent solutions which are called Jones modes. The space of all Jones modes corresponding to $\omega$ is denoted by $X_{\omega}\left(\Omega^{+}\right)$.

Theorem 1. Let $\overline{d_{1}} d_{2}$ be a negative real number and either $\Re(\bar{\beta} / \alpha)>0$ or $\alpha \beta=0$. Further, let $U$ and $w$ solve the homogeneous transmission problem (1)-(5) (i.e., $f_{j}=0, j=\overline{1,5}$ ). Then $w=0$ in $\Omega^{-}, u_{4}=0$ in $\Omega^{+}$, and $u=0$ in $\Omega^{+}$if $\omega \notin J\left(\Omega^{+}\right)$, and $u \in X_{\omega}\left(\Omega^{+}\right)$if $\omega \in J\left(\Omega^{+}\right)$.

Proof. Let a pair $(U, w)$ be a solution of the homogeneous Problem A. First we consider the case $\Re(\bar{\beta} / \alpha)>0$. Green's formula for the domain $\Omega^{+}$then 
implies [11]

$$
\begin{aligned}
\frac{2 \gamma}{i \omega \eta} \int_{\Omega^{+}}\left|\nabla u_{4}\right|^{2} d x= & \int_{S}\left(\{\bar{u}\}^{+}\{P U\}^{+}+\frac{\gamma}{i \omega \gamma}\left\{u_{4}\right\}^{+}\left\{\partial_{n} \bar{u}_{4}\right\}^{+}-\{u\}^{+}\{\overline{P U}\}^{+}\right. \\
& \left.+\frac{\gamma}{i \omega \eta}\left\{\overline{u_{4}}\right\}^{+}\left\{\partial_{n} u_{4}\right\}^{+}\right) d S
\end{aligned}
$$

where $\nabla=\left(\partial_{x_{1}}, \partial_{x_{2}}, \partial_{x_{3}}\right)$. From (10) and (3) it follows that

$$
\begin{aligned}
\frac{\gamma}{\omega \eta} \int_{\Omega^{+}}\left|\nabla u_{4}\right|^{2} d x= & -\Im\left(\bar{d}_{1} d_{2} \int_{S}\left\{\overline{\partial_{n} w}\right\}^{-}\{w\}^{-} d S\right) \\
& +\frac{\gamma}{\omega \eta} \Re \int_{S}\left\{u_{4}\right\}^{+}\left\{\overline{\partial u_{4}}\right\}^{+} d S .
\end{aligned}
$$

Further, let $B(0 ; R)=\left\{x \in \mathbb{R}_{3}:|x|<R\right\}$ be a ball with center at the origin and radius $R$. For sufficiently large $R$ we have $\Omega^{+} \subset B(0 ; R)$. Applying Green's formula for the operator $\Delta+\rho_{1} \omega^{2}$ and the domain $B(0 ; R) \backslash \overline{\Omega^{+}}$, we get

$$
\begin{aligned}
0= & \int_{B(0 ; R)}\left[w\left(\Delta+\rho_{1} \omega^{2}\right) \bar{w}-\bar{w}\left(\Delta+\rho_{1} \omega^{2}\right) w\right] d x \\
= & -\int_{S}\left(\{w\}^{-}\left\{\overline{\partial_{n} w}\right\}^{-}-\{\bar{w}\}^{-}\left\{\partial_{n} w\right\}^{-}\right) \\
& +\int_{\Sigma_{R}}\left(w \partial_{n} \bar{w}-\bar{w} \partial_{n} w\right) d \Sigma_{R},
\end{aligned}
$$

where $\left(\triangle+\rho_{1} \omega^{2}\right) w=0$ and $\Sigma_{R}=\partial B(0 ; R)$; the bar denotes complex conjugate. Equation (12) yields

$$
-\Im\left[\int_{S}\{w\}^{-}\left\{\partial_{n} w\right\}^{-} d S-\int_{\Sigma_{R}} w \partial_{n} \bar{w} d \Sigma_{R}\right]=0 .
$$

Multiplying (13) by $\overline{d_{1}} d_{2}<0$, adding termwise to (11), and passing to the limit as $R \rightarrow+\infty$ and taking (5) into consideration, we arrive at the equality

$$
\begin{array}{r}
\frac{\gamma}{\omega \eta}\left[\int_{\Omega^{+}}\left|\nabla u_{4}\right|^{2} d x+\Re\left(\frac{\bar{\beta}}{\alpha}\right) \int_{S}\left|u_{4}\right|^{2} d S\right] \\
-\omega \overline{d_{1}} d_{2} \sqrt{\varrho_{1}} \lim _{R \rightarrow+\infty} \int_{\Sigma_{R}}|w|^{2} d \Sigma_{R}=0 .
\end{array}
$$


Since all the constant multipliers involved in (14) are positive, from (14) we conclude that

$$
\begin{gathered}
\int_{\Omega^{+}}\left|\nabla u_{4}\right|^{2} d x+\Re\left(\frac{\bar{\beta}}{\alpha}\right) \int_{S}\left|u_{4}\right|^{2} d S=0, \\
\lim _{R \rightarrow+\infty} \int_{\Sigma_{R}}|w|^{2} d \Sigma_{R}=0 .
\end{gathered}
$$

Consequently, $u_{4}(x)=0$ in $\Omega^{+}$, and by Rellich's lemma (see, e.g, [2]) $w(x)=0$ in $\Omega^{-}$.

Now, let $\alpha=0$ and $\beta \neq 0$. By the same arguments as above we derive $u_{4}(x)=0$ in $\Omega^{+}$and $w(x)=0$ in $\Omega^{-}$.

If $\alpha \neq 0$ and $\beta=0$, then (14) yields $w(x)=0$ in $\Omega^{-}$, and $u_{4}(x)=$ const in $\Omega^{+}$, which in turn implies $u_{4}(x)=0$ in $\Omega^{+}$.

It is easy to see that, if $w=0$ in $\Omega^{-}$and $u_{4}(x)=0$ in $\Omega^{+}$, then $u$ solves the BVP (6)-(9), whence the proof of the theorem follows.

In accordance with Theorem 1 the uniqueness result for the Problem A is equivalent to the uniqueness result for the over-determined BVP (6)-(9). In what follows we shall show that the uniqueness result is essentially related to the geometrical shape of the interface surface $S$.

\section{Spherical Interfaces}

In this subsection we assume $\Omega^{+}=B(0, R), \partial \Omega^{+}=S=\Sigma_{R}, \overline{\Omega^{+}}=\Omega^{+} \bigcup S$, $\Omega^{-}=\mathbb{R}^{3} \backslash \overline{\Omega^{+}}$, and consider the homogeneous transmission Problem A.

In this case a general solution of system (1) can be represented in the form (for details see [13])

$$
\begin{aligned}
u & =a \operatorname{grad} \varphi_{1}+b \operatorname{grad} \varphi_{2}+c \operatorname{rot}\left\{[x \times \nabla] \varphi_{3}+\operatorname{rot}[x \times \nabla] \varphi_{4}\right\}, \\
u_{4} & =\varphi_{1}+\varphi_{2},
\end{aligned}
$$

where $\varphi_{j}$ are metaharmonic functions in $\Omega^{+}$,

$$
\left(\Delta+\omega_{j}^{2}\right) \varphi_{j}=0, \quad j=1,2,3,4,
$$

and where

$$
\begin{aligned}
a & =\frac{i(\lambda+2 \mu)\left(\varkappa \omega_{1}^{2}-i \omega\right)}{\varrho \omega^{3} \varkappa}+\frac{\gamma}{\varrho \omega^{2}}, \\
b & =\frac{i(\lambda+2 \mu)\left(\varkappa \omega_{2}^{2}-i \omega\right)}{\varrho \omega^{3} \eta \varkappa}+\frac{\gamma}{\varrho \omega^{2}}, \quad c=\frac{\mu}{\varrho \omega^{2}}, \\
\omega_{1}^{2} \omega_{2}^{2} & =\frac{i \varrho \omega^{3}}{\varkappa(\lambda+2 \mu)}, \quad \omega_{1}^{2}+\omega_{2}^{2}=\frac{i \omega}{\varkappa}+\frac{i \omega \gamma \eta}{\lambda+2 \mu}+\frac{\varrho \omega^{2}}{\lambda+2 \mu}, \\
\omega_{3}^{2} & =\omega_{4}^{2}=\frac{\varrho \omega^{2}}{\mu} .
\end{aligned}
$$


Clearly, $[x \times \nabla]_{k} \equiv \frac{\partial}{\partial S_{k}(x)}(k=1,2,3)$ represents a tangential derivative on the spherical surface of radius $|x|$ :

$$
\begin{aligned}
& \frac{\partial}{\partial S_{1}(x)}=x_{2} \frac{\partial}{\partial x_{3}}-x_{3} \frac{\partial}{\partial x_{2}}=-\cos \varphi \cot \theta \frac{\partial}{\partial \varphi}-\sin \varphi \frac{\partial}{\partial \theta}, \\
& \frac{\partial}{\partial S_{2}(x)}=x_{3} \frac{\partial}{\partial x_{1}}-x_{1} \frac{\partial}{\partial x_{3}}=-\sin \varphi \cot \theta \frac{\partial}{\partial \varphi}+\cos \varphi \frac{\partial}{\partial \theta}, \\
& \frac{\partial}{\partial S_{3}(x)}=x_{1} \frac{\partial}{\partial x_{2}}-x_{2} \frac{\partial}{\partial x_{1}}=\frac{\partial}{\partial \varphi} .
\end{aligned}
$$

Here $r, \theta, \varphi$ are the spherical co-ordinates of the point $x \in \mathbb{R}^{3}$.

Throughout this subsection we assume that

$$
\int_{\Sigma_{R}} \varphi_{3} d \Sigma=\int_{\Sigma_{R}} \varphi_{4} d \Sigma=0
$$

which guarantees the one-to-one correspondence between two sets of functions $u_{1}, \ldots, u_{4}$ and $\varphi_{1}, \ldots, \varphi_{4}$ (for details see [13]).

Lemma 2. If $\varphi_{1}, \ldots, \varphi_{4}$ are metaharmonic functions satisfying the Helmholtz equations (19), then $u=\left(u_{1}, u_{2}, u_{3}\right)^{\top}$ and $u_{4}$ defined by (17) and (18) solve the system of differential equations (1).

Vice versa, if a couple $\left(u, u_{4}\right)^{\top}$ is an arbitrary solution of the simultaneous equations (1), then there are uniquely defined metaharmonic functions $\varphi_{1}, \ldots, \varphi_{4}$ satisfying conditions (19) and (21) such that the representation formulas (17) and (18) hold true.

We look for functions $\varphi_{j}(j=\overline{1,4})$ and $w$ in the form (cf. [13])

$$
\begin{aligned}
\varphi_{j}(x) & =\sum_{m=0}^{\infty} \Psi_{m}\left(\omega_{j} r\right) Y_{j m}(\theta, \varphi), \quad j=\overline{1,4}, \quad x \in \Omega^{+}, \\
w(x) & =\sum_{m=0}^{\infty} \Phi_{m}\left(\omega_{5} r\right) Y_{5 m}(\theta, \varphi), \quad x \in \Omega^{-},
\end{aligned}
$$

where $Y_{l m}(\theta, \varphi)$ are spherical functions of order $m$,

$$
\begin{aligned}
\Psi_{m}\left(\omega_{j} r\right) & =\sqrt{\frac{R}{r}} \mathcal{I}_{m+\frac{1}{2}}\left(\omega_{j} r\right), \\
\Phi_{m}\left(\omega_{5} r\right) & =\sqrt{\frac{R}{r}} H_{m+\frac{1}{2}}^{(1)}\left(\omega_{5} r\right), \quad \omega_{5}=\sqrt{\rho_{1}} \omega,
\end{aligned}
$$

$\mathcal{I}_{m+\frac{1}{2}}$ and $H_{m+\frac{1}{2}}^{(1)}$ are Bessel function and Hankel function of first kind, respectively.

Moreover, we assume that $Y_{30}=Y_{40}=0$ by virtue of equations (21). In [13] it is shown that

$$
(x \cdot u)=a r \frac{\partial \varphi_{1}}{\partial r}+b r \frac{\partial \varphi_{2}}{\partial r}+c \sum_{k=1}^{3} \frac{\partial^{2} \varphi_{3}}{\partial S_{k}^{2}(x)},
$$




$$
\begin{aligned}
& (x \cdot P U)=\left\{2 a r \frac{\partial^{2}}{\partial r^{2}}-\left(a \lambda \omega_{1}^{2}+\gamma\right) r\right\} \varphi_{1}+\left\{2 b \mu r \frac{\partial^{2}}{\partial r^{2}}-\left(b \lambda \omega^{2}+\gamma\right) r\right\} \varphi_{2} \\
& +\frac{2 c \mu}{r}\left(r \frac{\partial}{\partial r}-1\right) \sum_{k=1}^{3} \frac{\partial^{2} \varphi_{3}}{\partial S_{k}^{2}(x)}, \\
& \sum_{k=1}^{3} \frac{\partial}{\partial S_{k}(x)}[x \times P U]_{k}=2 \mu\left(\frac{\partial}{\partial r}-\frac{1}{r}\right) \sum_{k=1}^{3} \frac{\partial^{2}}{\partial S_{k}^{2}}\left(a \varphi_{1}+b \varphi_{2}\right) \\
& -\left(2 c \mu r \frac{\partial^{2}}{\partial r^{2}}+2 c \mu \frac{\partial}{\partial r}-\frac{2 c \mu}{r}+\mu r\right) \sum_{k=1}^{3} \frac{\partial^{2} \varphi_{3}}{\partial S_{k}^{2}(x)}, \\
& \sum_{k=1}^{3} \frac{\partial}{\partial S_{k}(x)}[P U]_{k}=\mu\left(\frac{\partial}{\partial r}-\frac{1}{\partial r}\right) \sum_{k=1}^{3} \frac{\partial^{2} \varphi_{4}}{\partial S_{k}^{2}(x)} .
\end{aligned}
$$

Notice that (see [13])

$$
\sum_{k=1}^{3} \frac{\partial^{2} Z_{m}(\theta, \varphi)}{\partial S_{k}^{2}(x)}=-m(m+1) Z_{m}(\theta, \varphi),
$$

for an arbitrary spherical function $Z_{m}(\theta, \varphi)(m=\overline{0, \infty})$.

Substituting (22) into (25) and taking into account the homogeneous interface conditions (3) (i.e., $f_{j}=0, j=\overline{1,5}$ ) along with equation (26) and the orthogonality property of spherical functions, we obtain the system of linear algebraic equations for the above-introduced unknown spherical functions $Y_{j m}$ $(m=\overline{0, \infty}, j=\overline{1,5})$ :

$$
\begin{aligned}
a[ & \left.\frac{\partial}{\partial r} \Psi_{m}\left(\omega_{1} r\right)\right]_{r=R} Y_{1 m}+b\left[\frac{\partial}{\partial r} \Psi_{m}\left(\omega_{2} r\right)\right]_{r=R} Y_{2 m} \\
& -c m(m+1)\left[\frac{1}{R} \Psi_{m}\left(\omega_{3} R\right)\right] Y_{3 m}-d_{1}\left[\frac{\partial}{\partial r} \Phi_{m}\left(\omega_{5} r\right)\right]_{r=R} Y_{5 m}=0 \\
\{ & {\left.\left[2 a \mu \frac{\partial^{2}}{\partial r^{2}}-\left(a m \lambda \omega_{1}^{2}+\gamma\right)\right] \Psi_{m}\left(\omega_{1} r\right)\right\}_{r=R} Y_{1 m} } \\
& +\left\{\left[2 b \mu \frac{\partial^{2}}{\partial r^{2}}-\left(b \lambda \omega_{2}^{2}+\gamma\right)\right] \Psi_{m}\left(\omega_{2} r\right)\right\}_{r=R} Y_{2 m} \\
& -\left[\frac{2 c \mu}{r^{2}}\left(r \frac{\partial}{\partial r}-1\right) \Psi_{m}\left(\omega_{3} r\right)\right]_{r=R} m(m+1) Y_{3 m}-d_{2} \Phi_{m}\left(\omega_{5} R\right) Y_{5 m}=0 \\
& -2 \mu a\left[\frac{1}{r}\left(\frac{\partial}{\partial r}-\frac{1}{r}\right) \Psi_{m}\left(\omega_{1} r\right)\right]_{r=R} m(m+1) Y_{1 m} \\
& -2 \mu b\left[\frac{1}{r}\left(\frac{\partial}{\partial r}-\frac{1}{r}\right) \Psi_{m}\left(\omega_{2} r\right)\right]_{r=R} m(m+1) Y_{2 m} \\
& +m(m+1)\left[\left(2 c \mu \frac{\partial^{2}}{\partial r^{2}}+2 c \mu \frac{1}{r} \frac{\partial}{\partial r}-\frac{2 c \mu}{r^{2}}+\mu\right) \Psi_{m}\left(\omega_{3} r\right)\right]_{r=R} Y_{3 m}=0 \\
& -\mu m(m+1)\left[\left(\frac{\partial}{\partial r}-\frac{1}{r}\right) \Psi_{m}\left(\omega_{3} r\right)\right]_{r=R} Y_{4 m}=0
\end{aligned}
$$




$$
\left[\left(\alpha \frac{\partial}{\partial r}+\beta\right) \Psi_{m}\left(\omega_{1} r\right)\right]_{r=R} Y_{1 m}+\left[\left(\alpha \frac{\partial}{\partial r}+\beta\right) \Psi_{m}\left(\omega_{2} r\right)\right]_{r=R} Y_{2 m}=0 .
$$

Due to the uniqueness theorem we have $u_{4}=\varphi_{1}+\varphi_{2}=0$ in $\Omega^{+}$and $w=0$ in $\Omega^{-}$. Since $\omega_{1}^{2} \neq \omega_{2}^{2}$ for $\omega \neq 0$, it follows that $\varphi_{1}=\varphi_{2}=0$ in $\Omega^{+}$. Representations (22) and (23) then imply

$$
Y_{j m}(\theta, \varphi)=0, \quad j=1,2,5, \quad m=0,1,2, \ldots
$$

The first three equations in (27) now yield

$$
Y_{3 m}(\theta, \varphi)=0, \quad m=1,2, \ldots
$$

Thus the first, second, third and fifth equations in (27) are equivalent to the relations (28) and (29).

The fourth equation (for $m \geq 1$ ) can be rewritten in the form (see (24))

$$
\begin{aligned}
& {\left[\left(\frac{\partial}{\partial r}-\frac{1}{r}\right) \Psi_{m}\left(\omega_{3} r\right)\right]_{r=R} Y_{4 m}} \\
& \quad=\left[2 R \omega_{3} \mathcal{I}_{m+\frac{1}{2}}^{\prime}\left(\omega_{3} R\right)-3 \mathcal{I}_{m+\frac{1}{2}}\left(\omega_{3} R\right)\right] Y_{4 m}=0, \quad m=\overline{1, \infty} .
\end{aligned}
$$

The equation

$$
2 t \mathcal{I}_{m+\frac{1}{2}}^{\prime}(t)-3 \mathcal{I}_{m+\frac{1}{2}}(t)=0 \quad \text { with } \quad m \geq 1
$$

has infinitely many roots with respect to $t$ (see, e.g., [15]). Denote these roots by $\left\{t_{q}^{(m)}\right\}_{q=1}^{\infty}$ and let $Q:=\bigcup_{m=1}^{\infty}\left\{t_{q}^{(m)}\right\}_{q=1}^{\infty}$.

Further, let $\omega_{3} R=\omega R \sqrt{\frac{\varrho}{\mu}} \in Q$, i.e., $\omega R \sqrt{\frac{\varrho}{\mu}}=t_{q_{0}}^{\left(m_{0}\right)}$ for some $m_{0} \geq 1$ and $q_{0} \geq 1$. Then

$$
2 R \omega_{3} \mathcal{I}_{m_{0}+\frac{1}{2}}^{\prime}\left(\omega_{3} R\right)-3 \mathcal{I}_{m_{0}+\frac{1}{2}}\left(\omega_{3} R\right)=0
$$

and, consequently, equations (30) hold with arbitrary non-zero spherical functions $Y_{4 m_{0}}$ and $Y_{4 k}=0$ for $k \neq m_{0}$. It is evident that $\varphi_{4}(x):=\Psi_{m_{0}}(\omega r) Y_{4 m_{0}}(\theta, \varphi)$ is nontrivial and the vector

$$
u=\operatorname{rot} \operatorname{rot}[x \times \nabla] \varphi_{4}(x)=\omega_{3}^{2}[x \times \nabla] \varphi_{4}(x)
$$

represents a nontrivial solution of problem (6)-(9). Therefore $\omega=\sqrt{\frac{\mu}{\varrho}} \frac{t_{q_{0}}^{\left(m_{0}\right)}}{R}$ is a Jones frequency and (32) is the corresponding Jones mode. The above results lead to the following assertion.

Theorem 3. Let $\Omega^{+}=B(0, R)$ and

$$
\begin{aligned}
& \left(\Delta+\omega_{3}^{2}\right) \varphi_{4}(x)=0 \quad \text { in } \quad \Omega^{+} \quad \text { with } \quad \omega_{3} R=t_{q_{0}}^{\left(m_{0}\right)} \in Q, \\
& {\left[\left(\frac{\partial}{\partial r}-\frac{1}{r}\right) \varphi_{4}(x)\right]_{r=R}=0 \quad \text { for } \quad|x|=R,} \\
& {[x \times \nabla] \varphi_{4}(x) \not \equiv 0 .}
\end{aligned}
$$

Then $\omega$ is a Jones frequency and the vector

$$
u(x)=\omega_{3}^{2}[x \times \nabla] \varphi_{4}(x), \quad x \in B(0, R)
$$

is a Jones mode. 
Vice versa, if $\omega$ is a Jones frequency and $u$ is a Jones mode, then there exists $\varphi_{4}$ such that (34) holds and $\varphi_{4}$ solves the BVP (33) with some $\omega_{3}$ such that $\omega_{3} R \in Q$.

From Theorem 3 it follows immediately that the set of Jones eigenfrequencies is given by the relation

$$
J\left(\Omega^{+}\right)=\left\{\omega: \omega=\sqrt{\frac{\mu}{\varrho}} \frac{t_{q}^{(m)}}{R} \quad \text { with } \quad t_{q}^{(m)} \in Q\right\} .
$$

Applying the properties of spherical functions it is easy to show that to each eigenfrequency $\omega=\sqrt{\frac{\mu}{\varrho}} \frac{t_{q}^{(m)}}{R}$ there correspond $2 m+1$ linearly independent Jones modes.

\section{Interfaces with Flat Sub-Manifolds}

Here we describe a class of domains with a special geometry for which there exist no Jones modes.

Let $\Omega^{+}$be a bounded domain with a piecewise $C^{2}$-smooth, simply connected Lipschitz boundary $S=\partial \Omega^{+}$and consider the homogeneous transmission problem (1)-(5).

Since, in view of Theorem $1, w=0$ in $\Omega^{-}$and $u_{4}=0$ in $\Omega^{+}$, we easily derive that $u=\left(u_{1}, u_{2}, u_{3}\right)^{\top}$ is a solution to the over-determined BVP (6)-(9).

In what follows we assume that the displacement vector $u=\left(u_{1}, u_{2}, u_{3}\right)^{\top}$ belongs to the Sobolev space $W_{2}^{1}\left(\Omega^{+}\right)$. Therefore, due to the well-known regularity theorems for weak solutions, we have

$$
u \in W_{2}^{1}\left(\Omega^{+}\right) \cap C^{\infty}\left(\Omega^{+}\right) \cap C^{2}\left(\Omega^{+} \cup S^{0}\right),
$$

where $S^{0}$ is the union of $C^{2}$-smooth open sub-manifolds of $S$. We note that if some open sub-manifold $S^{*} \subset S$ is $C^{\infty}$-smooth, then $u \in C^{\infty}\left(\Omega^{+} \cup S^{*}\right)$.

Further, we recall that for the components $u_{j}(j=1,2,3)$ of the displacement vector $u$, which are solutions of the Helmholtz equation (6) in the domain $\Omega^{+}$, there hold the following integral representation formulae (see, e.g., [2])

$$
\begin{gathered}
\int_{S}\left\{\left[\partial_{n(y)} \gamma(x-y, \omega)\right]\left[u_{j}(y)\right]^{+}-\gamma(x-y, \omega)\left[\partial_{n} u_{j}(y)\right]^{+}\right\} d S_{y} \\
=\left\{\begin{array}{lll}
u_{j}(x) & \text { for } & x \in \Omega^{+}, \\
0 & \text { for } & x \in \Omega^{-},
\end{array}\right.
\end{gathered}
$$

where $\gamma(x, \omega)=-(4 \pi|x|)^{-1} \exp \left\{i \omega_{3}|x|\right\}$ is a fundamental function of the Helmholtz operator $\Delta+\omega_{3}^{2}$ with $\omega_{3}=\omega \sqrt{\frac{\varrho}{\mu}}$.

Theorem 4. Let the boundary surface $S=\partial \Omega^{+}$be a Lipschitz piecewise smooth manifold which contains two flat, open sub-manifolds $S_{1}$ and $S_{2}$ with non-parallel normal vectors.

Then the homogeneous BVP (6)-(9) possesses only a trivial solution. 
Proof. Denote by $a^{(1)}=\left(a_{11}, a_{12}, a_{13}\right)$ and $a^{(2)}=\left(a_{21}, a_{22}, a_{23}\right)$ the outward unit normal vectors to $S_{1}$ and $S_{2}$, respectively. Clearly, $a_{p j}(p=1,2, j=1,2,3)$ are real constants and due to the assumptions of the theorem

$$
a:=a^{(1)} \times a^{(2)}=\left(a_{1}, a_{2}, a_{3}\right) \neq 0 .
$$

Further, we introduce the metaharmonic functions in $\Omega^{+}$, related to the displacement vector $u$ as follows:

$$
v_{p}(x)=u(x) \cdot a^{(p)}=a_{p 1} u_{1}(x)+a_{p 2} u_{2}(x)+a_{p 3} u_{3}(x), \quad p=1,2 .
$$

Obviously,

$$
v_{p} \in W_{2}^{1}\left(\Omega^{+}\right) \cap C^{\infty}\left(\Omega^{+} \cup S_{1} \cup S_{2}\right) \cap C^{2}\left(\Omega^{+} \cup S^{0}\right),
$$

where $S^{0}$ is the same as above. It is also evident that the representation formula (35) remains valid for $v_{p}(p=1,2)$.

Let us show that the function $v_{p}(p=1,2)$ vanishes in $\Omega^{+}$. To this end, for definiteness, we put $p=1$ and rewrite the boundary conditions (8) as

$$
\left\{2 \partial_{n} u+[n \times \operatorname{curl} u]\right\}_{q}^{+}=\left\{\partial_{n} u_{q}+\sum_{j=1}^{3} n_{j} \partial_{q} u_{j}\right\}^{+}=0 \quad \text { on } \quad S, \quad q=1,2,3 .
$$

These equations imply

$$
\left\{\partial_{n} u_{q}+\partial_{q}\left(\sum_{j=1}^{3} a_{1 j} u_{j}\right)\right\}^{+}=0 \quad \text { on } \quad S_{1}, \quad q=1,2,3,
$$

since $n_{j}=a_{1 j}$ on $S_{1}$.

By multiplying the $q$-th equation by $n_{q}=a_{1 q}$ and summing, we get

$$
\left\{\partial_{n}\left(\sum_{q=1}^{3} a_{1 q} u_{q}\right)+\partial_{n}\left(\sum_{j=1}^{3} a_{1 j} u_{j}\right)\right\}^{+}=0 \quad \text { on } S_{1}
$$

i.e.,

$$
\left\{\partial_{n} v_{1}(x)\right\}^{+}=0 \quad \text { on } \quad S_{1} .
$$

Moreover, by the boundary condition (9) we have

$$
\left\{v_{1}(x)\right\}^{+}=0 \text { on } S_{1} .
$$

Therefore, due to the integral representation formula (35), we arrive at the equation

$$
\begin{gathered}
\int_{S \backslash S_{1}}\left\{\left[\partial_{n(y)} \gamma(x-y, \omega)\right]\left[v_{1}(y)\right]^{+}-\gamma(x-y, \omega)\left[\partial_{n} v_{1}(y)\right]^{+}\right\} d S_{y} \\
=\left\{\begin{array}{lll}
v_{1}(x) & \text { for } & x \in \Omega^{+} \\
0 & \text { for } & x \in \Omega^{-} .
\end{array}\right.
\end{gathered}
$$

Taking into account that the left-hand side integral in (39) defines an analytic function of the real variable $x$ in the connected domain $\mathbb{R}^{3} \backslash \overline{S_{1}}$ which vanishes in $\Omega^{-} \subset \mathbb{R}^{3} \backslash \overline{S_{1}}$, we conclude that this integral is zero in the whole domain of 
analyticity $\mathbb{R}^{3} \backslash \overline{S_{1}}$. Hence $v_{1}(x)=0$ in $\Omega^{+}$. By the same arguments we can show that $v_{2}(x)=0$ in $\Omega^{+}$.

Now from (37) we see that the displacement vector $u(x)$ for arbitrary $x \in \Omega^{+}$ is parallel to the constant vector $a$ given by (36). Therefore there exists a scalar function $v(x)$ such that

$$
u(x)=a v(x), \quad \text { i.e., } \quad u_{j}(x)=a_{j} v(x) \quad \text { for } \quad x \in \Omega^{+}, \quad j=1,2,3 .
$$

It is easy to check that the function $v$ satisfies the conditions (cf. (6), (8), (9), $(38))$ :

$$
\begin{aligned}
& \left(\Delta+\omega_{3}^{2}\right) v(x)=0 \quad \text { in } \Omega^{+}, \\
& (a \cdot n(x))\{v(x)\}^{+}=0 \text { on } S, \\
& \left\{a_{q} \partial_{n} v(x)+\sum_{j=1}^{3} n_{j} a_{j} \partial_{q} v(x)\right\}^{+}=0 \quad \text { on } \quad S, \quad q=1,2,3 .
\end{aligned}
$$

The last two equations yield

$$
b(x)\{v(x)\}^{+}=0 \quad \text { and } \quad b(x)\left\{\partial_{n} v(x)\right\}^{+}=0 \quad \text { on } \quad S,
$$

where

$$
b(x)=a \cdot n(x)=a_{1} n_{1}(x)+a_{2} n_{2}(x)+a_{3} n_{3}(x), \quad x \in S,
$$

with $n(x)$ being the outward unit normal to $S$ and with $a$ given by (36).

Since the vector $a$ is constant and $S$ is a piecewise $C^{2}$-smooth closed surface it follows that there exists an open $C^{2}$-smooth sub-manifold $S^{*} \subset S$ such that

$$
b(x) \neq 0 \text { for } x \in S^{*} .
$$

Therefore (41) implies

$$
\{v(x)\}^{+}=0 \quad \text { and } \quad\left\{\partial_{n} v(x)\right\}^{+}=0 \quad \text { on } \quad S^{*},
$$

whence, by the same arguments as above, we deduce that $v(x)=0$ in $\Omega^{+}$. Finally, equations (40) show that the displacement vector $u(x)$ vanishes in $\Omega^{+}$.

\section{REFERENCES}

1. A. Boström, Scattering of stationary acoustic waves by an elastic obstacle immersed in a fluid. J. Acoust. Soc. Amer. 67(1980), No. 2, 390-398.

2. D. Colton and R. Kress, Integral equation methods in scattering theory. Pure and Applied Mathematics (New York). A Wiley-Interscience Publication. John Wiley \& Sons, Inc., New York, 1983.

3. G. C. Everstine and M. K. Au-YAng, eds., Advances in Fluid-Structure Interaction. American Society of Mechanical Engineers, New York, 1984.

4. G. C. Hsiao, R. E. Kleinman, and G. F. Roach, Weak solution of fluid-solid interaction problems. Technische Hochschule Darmstadt, Fachbereich Mathematik, Preprit Nr. 1917, 1997.

5. L. Jentsch and D. NAtroshvili, Interaction between thermoelastic and scalar oscillation fields. Integral Equations Operator Theory 28(1997), No. 3, 261-288. 
6. L. Jentsch and D. Natroshvili, Non-local approach in mathematical problems of fluid-structure interaction. Math. Methods Appl. Sci. 22(1999), No. 1, 13-42.

7. L. Jentsch and D. NAtroshvili, Three-dimensional mathematical problems of thermoelasticity of anisotropic bodies. I. Mem. Differential Equations Math. Phys. 17(1999), $7-126$.

8. L. Jentsch and D. NAtroshvili, Three-dimensional mathematical problems of thermoelasticity of anisotropic bodies. II. Mem. Differential Equations Math. Phys. 18(1999), $1-50$.

9. D. S. Jones, Low-frequency scattering by a body in lubricated contact. Quart. J. Mech. Appl. Math. 36(1983), No. 1, 111-138.

10. M. C. Junger and D. Fiet, Sound, structures and their interaction. MIT Press, Cambridge, MA, 1986.

11. V. D. Kupradze, T. G. Gegelia, M. O. Basheleishvili, and T. V. Burchuladze, Three-dimensional problems of the mathematical theory of elasticity and thermoelasticity. (Russian) Nauka, Moscow, 1976; English transl.: North-Holland Series in Applied Mathematics and Mechanics, 25. North-Holland Publishing Co., Amsterdam-New York, 1979.

12. C. J. Luke and P. A. Martin, Fluid-solid interaction: acoustic scattering by a smooth elastic obstacle. SIAM J. Appl. Math. 55(1995), No. 4, 904-922.

13. D. Natroshvili and M. Svanadze, Some dynamic problems of coupled thermoelasticity for piecewise-homogeneous bodies. (Russian) Tbiliss. Gos. Univ. Inst. Prikl. Mat. Trudy 10(1981), 99-190.

14. D. Natroshvili and G. Sadunishvili, Interaction of elastic and scalar fields. Math. Methods Appl. Sci. 19(1996), No. 18, 1445-1469.

15. A. F. Nikiforov and V. B. Uvarov, Special functions of mathematical physics. Nauka, Moscow, 1978.

16. W. Nowacki, Dynamic problems of thermoelasticity. PWN-Polish Scientific Publishers, Warszawa, Poland, Nordhoff International Publishing, Leyden, The Nederlands, 1975.

17. R. Ohayon and E. Sanchez-Palencia, On the vibration problem for an elastic body surrounded by a slightly compressible fluid. RAIRO Anal. Numér. 17(1983), No. 3, 311326.

(Received 01.12.2004)

Authors' address:

Department of Mathematics

Georgian Technical University

77, M. Kostava St., Tbilisi 0175

Georgia

E-mail: natrosh@hotmail.com 